\title{
The COVID-19 pandemic reduced the trauma incidence and modified its pattern in Al-Ain City, United Arab Emirates
}

\author{
David Olukolade Alao ${ }^{1,2}$ (D) Arif Alper Cevik ${ }^{1,2} \cdot$ Yasin Jemal Yasin $^{3} \cdot$ Thiagarajan Jaiganesh $^{2} \cdot$ Fikri Abu-Zidan $^{4}$
}

Received: 11 November 2021 / Accepted: 30 January 2022 / Published online: 18 February 2022

(c) The Author(s) 2022

\begin{abstract}
Aim We aimed to study the impact of the COVID-19 pandemic on the pattern of injury and outcome of hospitalized trauma patients in Al-Ain City, United Arab Emirates, to use this information in the preparedness for future pandemics.

Methods We retrospectively compared the trauma registry data of all hospitalised trauma patients, who were treated at the two main trauma centres in Al-Ain City (Al-Ain Hospital and Tawam Hospital); those who were treated over 1 year before the pandemic $(n=2002)$ and those who were treated at the first year of the COVID-19 pandemic $(n=1468)$.

Results There was a $26.7 \%$ reduction in the overall incidence of trauma admissions in the COVID-19 pandemic period. The mechanism of injury significantly differed between the two periods ( $p<0.0001$, Fisher's exact test). There was an absolute increase in the number of injuries, due to machinery and falling objects during the pandemic $(39.7 \%$ and $54.1 \%$ respectively, $p<0.001)$. In contrast, road traffic collisions and falls were reduced by $33.5 \%$ and $31.3 \%$, respectively. Location significantly differed between the two periods ( $p<0.0001$, Fisher's exact test). There was an absolute increase of $18.4 \%$ in workplace injuries and a reduction of $39.3 \%$ in home injuries over the study period. In addition, we observed relatively more workplace injuries and fewer home injuries during the pandemic (11.3\% and $42.8 \%$ compared with $7.1 \%$ and $52.4 \%$, respectively). Mortality was similar between the two periods ( $1.8 \%$ compared with $1.2 \%, p=0.16$, Fisher's exact test).

Conclusions The COVID-19 pandemic has modified the trauma risk exposure in our population. It reduced trauma hospital admissions by around $27 \%$. Work-related injuries, including falling objects and machinery injuries, were relatively higher during the pandemic. Prevention of work-related injuries should be an important component of preparedness for future pandemics.
\end{abstract}

Keywords COVID-19 · Trauma · Injury · Epidemiology · Death · United Arab Emirates

\begin{tabular}{|c|c|c|c|}
\hline Abbreviations & & RT-PCR & $\begin{array}{l}\text { Reverse transcriptase-polymerase chain } \\
\text { reaction }\end{array}$ \\
\hline DOH & Department of health & & \\
\hline GCS & Glasgow coma scale & SARS-COV-19 & Severe acute respiratory syndrome coro- \\
\hline ICU & Intensive care unit & & nal virus-2019 \\
\hline ISS & Injury severity score & UAE & United Arab Emirates \\
\hline RTC & Road traffic collision & WHO & World Health Organization \\
\hline
\end{tabular}

David Olukolade Alao

davidalao@uaeu.ac.ae

Arif Alper Cevik

aacevik@uaeu.ac.ae

Yasin Jemal Yasin

201990037@uaue.ac.ae

Thiagarajan Jaiganesh

tjaiganesh@seha.ae

Fikri Abu-Zidan

fabuzidan@uaeu.ac.ae
1 Department of Internal Medicine, College of Medicine and Health Sciences, UAE University, Al-Ain, United Arab Emirates

2 Emergency Department, Tawam Hospital, Al-Ain, United Arab Emirates

3 Institute of Public Health, College of Medicine and Health Sciences, UAE University, Al-Ain, United Arab Emirates

4 Department of Surgery, College of Medicine and Health Sciences, UAE University, Al-Ain, United Arab Emirates 


\section{Introduction}

Major trauma accounts for 5.8 million deaths annually and is the leading cause of death among children and young adults below 44 years [1]. Trauma is the second leading cause of death in the United Arab Emirates (UAE) behind cardio-vascular diseases. In 2017, it accounted for $17.2 \%$ of all deaths in the UAE, with motor vehicle collisions and falls from a height constituting over $70 \%$ of these deaths [2].

The COVID-19 pandemic started in 2019 in Wuhan, China. On 11th March 2020, the WHO declared it a worldwide pandemic. This has a significant impact on healthcare provision and resource utilisation, including trauma care. The first case of COVID-19 was confirmed in the UAE on 29th January 2020. By March 2020, several thousands of people had been infected by the virus. This necessitated a declaration of total lockdown by the UAE government.

Studies across the globe have reported a significant reduction in the incidence of trauma during the COVID-19 pandemic [3-6]. The lockdown imposed by different governments has reduced motor vehicle collision injuries, while interpersonal violence has increased due to more direct contact at home. We have shown recently that the COVID-19 lockdown reduced the road traffic collision (RTC) incidence worldwide, while increasing its severity. This was attributed to empty lanes, increased speed, and drivers ignoring traffic regulations [7, 8]. We anticipated that the COVID-19 pandemic impact on trauma outcomes might be different in our population. We aimed to study the impact of the COVID-19 pandemic on the pattern of injury and outcome of hospitalized trauma patients in Al-Ain City, UAE, to inform the preparedness for future pandemics.

\section{Patients and methods}

\section{Ethical consideration}

The Department of Health, Abu Dhabi Institutional Review Body, gave ethical approval for this study (Ref: DOH/ CVDC/2021/650). The Al-Ain Hospital and Tawam Hospital institutional review body approved the study.

\section{Setting}

Al-Ain Region of Abu Dhabi, United Arab Emirates, has an estimated population of 766,000. It had two major hospitals that received trauma emergencies prior to the pandemic. On 28th March 2020, following the outbreak of COVID19, the hospitals were redesignated as COVID-19 and nonCOVID-19. Al-Ain Hospital, which received the majority of the trauma patients before the outbreak, became the COVID19 hospital. In contrast, Tawam Hospital was designated a non-COVID hospital and the only trauma receiving hospital. All trauma patients either self-presented or were brought by the emergency medical service to Tawam Hospital. The patients were managed and stabilised in the emergency department until the result of their SARS-COV-19 reverse transcriptase-polymerase chain reaction (RT PCR) test was known. Patients with a positive result were transferred to the COVID-19 hospital for ongoing care. If a patient needed an emergency life-saving operation, they would be operated on without the PCR test and kept in the recovery room of the operating theatre until the PCR result was received. This would take around $4 \mathrm{~h}$. Patients would be admitted to Tawam Hospital if the result was negative; otherwise, they would be transferred to Al-Ain Hospital for further management.

\section{Patients}

We studied all trauma patients who died in the hospital or who were admitted for more than $24 \mathrm{~h}$ at both Al-Ain and Tawam Hospital from 28th March 2019 to 27th March 2020 (pre-pandemic period) and those who were admitted to Tawam Hospital from 28th March 2020 to 27th March 2021 (pandemic period).

\section{Data collection}

We extracted, from the Abu Dhabi trauma registry, anonymised data of all trauma patients who were admitted and treated at Tawam and Al-Ain Hospital over the study period. The Abu Dhabi trauma registry started in 2013 and was based on the national trauma data bank of the American College of Surgeons. It prospectively collects data on all trauma patients who died in the hospital or had been admitted for more than $24 \mathrm{~h}$.

\section{Studied variables}

We compared the two periods regarding patients' demographics, mechanisms of injury, locations of injury, physiological and anatomical severity indicators, ISS, hospital and ICU length of stay, and clinical outcome, including death.

\section{Statistical analysis}

Since the population of $\mathrm{Al}$ Ain did not change over the study periods, the absolute numbers would indirectly reflect the incidence of each mechanism of injury. Accordingly, changes between the two periods were reported in both absolute and relative values. Data are presented as number (\%) for categorical data, median (range) for ordinal data and mean (standard deviation) for continuous data. We used 
Fisher's exact test to compare categorical data of two independent groups, while the Mann-Whitney $U$ test was used for continuous or ordinal data of two independent groups. Statistical Package for the Social Sciences (IBM-SPSS version 26, Chicago, Il) was used for all analyses. A $p$ value of less than 0.05 was accepted as significant.

\section{Results}

Table 1 shows the patients' demographics and physiological parameters for the two periods. A total of 2002 trauma patients (79.9\% males) were admitted in the pre-COVID period, representing an annual trauma incidence of 261.4 trauma patients per 100,000 population. In the second period, a total of 1468 (78.7\% male) trauma patients were admitted representing an incidence rate of 191.6/100,000 population and a drop of $26.7 \%$ in annual incidence rate. Three patients in the second period tested positive for COVID-19 and were transferred to Al Ain Hospital. All survived to discharge. Figure 1 shows the maximum reduction from March to July 2020 when the government imposed a total lockdown, except for essential services, in the country. There was a rise in rates as the restrictions were lifted during August 2020.

Table 1 Demography of hospitalized trauma patients during the prepandemic period and the pandemic period, Al-Ain City, United Arab Emirates

\begin{tabular}{lccc}
\hline Variable & $\begin{array}{l}\text { Pre-COVID } \\
n=2002\end{array}$ & $\begin{array}{l}\text { COVID } \\
n=1468\end{array}$ & $p$ value \\
\hline Age & $28(0-104)$ & $29(1-96)$ & 0.17 \\
Gender & & & \\
Male & $1600(79.9)$ & $1154(78.7)$ & 0.37 \\
Female & $402(20.1)$ & $313(21.3)$ & \\
Nationality & & & $<0.001$ \\
UAE nationals & $881(44.7)$ & $545(38)$ & \\
Non-UAE & $1089(55.3)$ & $891(62)$ & \\
SBP & $132(0-251)$ & $127(0-215)$ & $<0.001$ \\
Heart rate & $89(0-199)$ & $91(0-189)$ & 0.51 \\
Respiratory rate & $18(0-60)$ & $20(0-48)$ & $<0.001$ \\
GCS* & $15(3-15)$ & $15(3-15)$ & 0.38 \\
& $14.6(1.8)$ & $14.6(1.8)$ & \\
ISS* & $4(1-75)$ & $4(1-38)$ & $<0.001$ \\
& $6.3(6.2)$ & $5.6(5.5)$ & \\
ISS $>15$ & $118(5.9)$ & $87(5.9)$ & 0.99 \\
Hospital days & $2(1-157)$ & $2(1-93)$ & 0.79 \\
ICU admit & $151(7.5)$ & $131(8.9)$ & 0.15 \\
Death & $25(1.2)$ & $27(1.8)$ & 0.16 \\
\hline
\end{tabular}

Data are presented as median (range) and mean (SD). $p$ value Fisher's exact test or Mann-Whitney test as appropriate

*GCS and *ISS are presented both as median (range) and mean (SD)

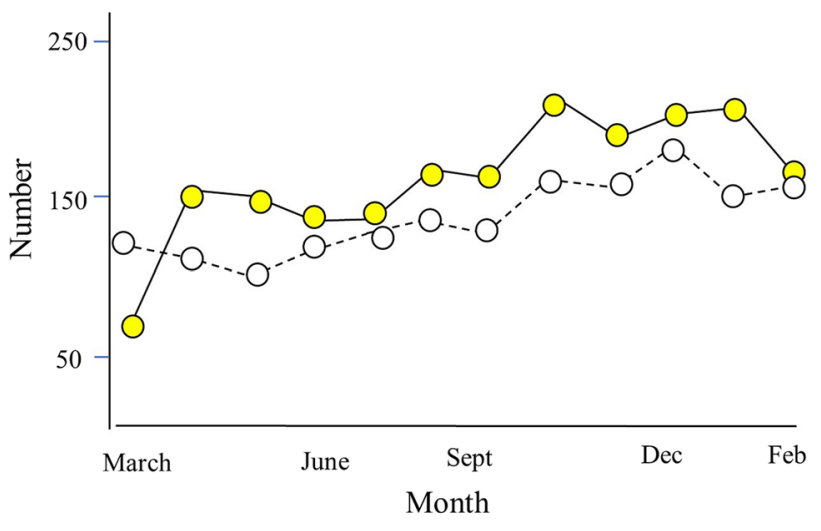

Fig. 1 Hospitalised trauma patients during the pre-pandemic period (yellow circles) and pandemic period (white circles), Al-Ain City, United Arab Emirates

Significantly fewer UAE nationals were admitted during the COVID-19 period (38\% compared with $44.7 \%$, $p<0.001$ ); an absolute reduction of $38 \%$. Patients admitted during the COVID-19 period had significantly lower SBP, higher respiratory rates and lower ISS $(p<0.001)$. There was no significant difference in the hospital length of stay $(p=0.79)$, nor in the overall mortality rates between the two periods $(1.2 \%$ compared with $1.8 \%, p=0.16$, Fisher's exact test).

Mechanism of injury significantly differed between the two periods $(p<0.0001$, Fisher's exact test). The absolute number of injuries due to machinery and falling objects increased in the pandemic period $(39.4 \%$ and $54.1 \%$, respectively). In contrast, RTCs and falls were reduced during the pandemic period (33.5\% and 31.3\%, respectively). Injury due to machinery and falling objects was relatively higher in the pandemic period $(7.5 \%$ and $3.9 \%$ compared with $3.9 \%$ and $1.8 \%$, respectively), while RTCs and falls were relatively less during the pandemic period (34.3\% and 36\% compared with $37.5 \%$ and $38.1 \%$, respectively) (Table 2). Table 3 shows the locations of injury. Location significantly differed between the two periods ( $p<0.0001$, Fisher's exact test). There was an absolute increase of $18.4 \%$ in workplace injuries and a reduction of $39.3 \%$ in home injuries over the study period. There were relatively more workplace injuries and relatively fewer home injuries during the pandemic period (11.3\% and $42.8 \%$ compared with $7.1 \%$ and $52.4 \%$, respectively).

\section{Discussion}

This study has shown a $27 \%$ reduction in the overall incidence of trauma admissions during the COVID-19 pandemic. Work-related injuries, including falling objects and machinery injuries, were absolutely and relatively more 
Table 2 Mechanism of injury of hospitalized trauma patients during the pre-pandemic period and pandemic period, Al-Ain City, United Arab Emirates

\begin{tabular}{lccl}
\hline Mechanism & $\begin{array}{l}\text { Pre-COVID } \\
n=2002\end{array}$ & $\begin{array}{l}\text { COVID } \\
n=1455\end{array}$ & Change (\%) \\
\hline Falls & $763(38.1)$ & $524(36)$ & -31.3 \\
RTC & $750(37.5)$ & $499(34.3)$ & -33.5 \\
Penetrating & $70(3.5)$ & $70(4.8)$ & 0 \\
Machinery & $78(3.9)$ & $109(7.5)$ & +39.7 \\
Burns & $46(2.3)$ & $37(2.5)$ & -19.6 \\
Assault & $55(2.7)$ & $52(3.6)$ & -5.5 \\
Falling objects & $37(1.8)$ & $57(3.9)$ & +54.1 \\
Others & $203(10.1)$ & $107(7.4)$ & -47.3 \\
\hline
\end{tabular}

Data are presented as number $(\%)$, the mark of percentage change indicates an increase $(+)$ or decrease $(-)$. Numbers do not add to the total number of the population because of missing data. Percentages were calculated from available data

Table 3 Location of injury of hospitalized trauma patients during the pre-pandemic period and the pandemic period, Al-Ain City, United Arab Emirates

\begin{tabular}{lccc}
\hline Location* & $\begin{array}{l}\text { Pre-COVID } \\
n=1913\end{array}$ & $\begin{array}{l}\text { COVID } \\
n=1424\end{array}$ & $p$ value \\
\hline Home & $1003(52.4)$ & $609(42.8)$ & $<0.001$ \\
Highway/street & $545(28.5)$ & $434(30.5)$ & \\
Workplace/farm & $136(7.1)$ & $161(11.3)$ & \\
Public area & $61(3.2)$ & $10(0.7)$ & \\
Others & $168(8.8)$ & $210(14.7)$ & \\
\hline
\end{tabular}

Data are presented as number (\%)

* Numbers do not add to the total number of the population because of missing data. Percentages were calculated from available data

during the pandemic, while RTCs and fall injuries were absolutely and relatively less. There were more workplace injuries and fewer home injuries during the pandemic period. Furthermore, fewer UAE nationals were admitted during the pandemic. However, there was no significant difference in mortality between the two periods.

UAE nationals represent $44.7 \%$ of the total trauma admissions in the pre-pandemic period, although they constitute $30 \%$ of the population in Al-Ain City [9]. This has been attributed to the difference in risk exposure and behaviour between UAE nationals and non-nationals, mainly in RTCs [10]. During the pandemic period, trauma admissions of UAE nationals reduced by $38.1 \%$. Most of the UAE nationals are governmental office employees, who were asked to work from home, compared with non-nationals who ran basic services and essential work outside homes. Although more people worked from home during the pandemic, there was around a $39 \%$ reduction in the number of injured patients that occurred at home. We have previously shown that $39 \%$ of trauma admissions in our setting were due to injuries that occurred at home, and that falls on the same level in the elderly and UAE nationals have increased over the last decade [11, 12]. The "stay-at-home" order during the pandemic may have increased the level of supervision for those at risk of falls. The relative increase in work-related injuries from machinery and falling objects are related to increased injuries in non-national workers. Similar to our results, other studies have reported differences in trauma patterns by race and gender during the COVID pandemic [13-15].

Over the study periods, the number of injured patients from RTCs and falls reduced by $33.5 \%$ and $31.3 \%$, respectively. This reflects the reduction in person and vehicular movements during the pandemic, especially during March-July 2020 when there was a total lockdown in the Abu Dhabi Emirate with the closure of public facilities and compulsory "stay-at-home" orders by all except essential workers [16]. Many countries worldwide have reported a similar reduction in trauma burden from RTCs and outdoors activities ranging from 20.3 to $84 \%$ [17-20].

The two leading causes of trauma deaths in our setting are RTCs (47\%) and work-related falls from height [9]. Both of these mechanisms of injury were reduced during the pandemic, while mortality did not change. Although some countries have reported a reduction in trauma mortality rate during the pandemic, others have reported an increase [21]. This may be related to a rise in vehicle speeding and noncompliance with safety rules in the pandemic period. An increase in interpersonal injuries and deliberate self-harm during the pandemic were reported [22, 23]. This could be related to the lockdown of the abusers with their victims and the reluctance of the victims to leave their homes due to the COVID-19 risk [24]. These injuries represent less than $4 \%$ at both periods in our study, with no significant change in trend. This is possibly related to supportive family ties and a low incidence of violence in our community.

\section{Limitations}

We have to acknowledge that our study has certain limitations. First, we studied hospitalised trauma patients only in Al-Ain City and not on a national level, which may limit the generalisability of our study to the whole of the UAE. Nevertheless, the laws and regulations during the lockdown were applied nationally, and we think these results may be extrapolated to other cities within the UAE. Second, we may have underestimated the trauma death rates because our registry excludes those who died on the scene. Third, we have to highlight that the sample sizes of each period are relatively small. Despite having lower ISS in the COVID-19 period, mortality was higher (although non-significant). This can be attributed to a delay 
in the management of trauma patients waiting for the PCR test before admission. This is supported by the fact that those having an ISS of more than 16 were the same between the two periods. Finally, the reduction of trauma incidence over time can be part of the trend of overall improvement in our trauma system, including injury prevention. Mortality of those having severe trauma has dramatically improved over time in our setting [11]. Nevertheless, we were able to demonstrate an immediate rebound increase in trauma following maximum lockdown supports our conclusions.

\section{Conclusions}

The COVID-19 pandemic has modified the trauma risk exposure in our population. It reduced trauma hospital admissions by around $27 \%$. Work-related injuries, including falling objects and machinery injuries were relatively higher during the pandemic. Injury prevention of work-related injuries should be an important component of preparedness for future pandemics.

Acknowledgements The authors thank Virgie Guy Pedo, Medical Research Specialist, Department of Internal Medicine, College of Medicine and Health Sciences, for her technical assistance in data coding.

Author contributions DOA, TJ, AAC and FMAZ contributed to the study's conception and design. DOA, TJ, YJY and FMAZ contributed to the acquisition and coding of data. AAC and FMAZ analysed the data. DOA wrote the paper. FMAZ, AAC, and TJ critically read the manuscript. All authors read and approved the final manuscript.

Funding There was no funding for this research study.

Data availability There are no additional data available to share with the readers. Data will be shared with the Editor if requested.

\section{Declarations}

Conflict of interest The authors declare that they have no conflict of interest.

Ethical approval and consent to participate Ethical approval for this study was obtained from the Abu Dhabi Health Research and Technology Ethics Committee, the Department of Health, Abu Dhabi, United Arab Emirates (Ref: DOH/CVDC/2021/650). Written informed consent was taken from the patients or their caregivers to use patient data in this research study.

Consent for publication Not applicable.

Open Access This article is licensed under a Creative Commons Attribution 4.0 International License, which permits use, sharing, adaptation, distribution and reproduction in any medium or format, as long as you give appropriate credit to the original author(s) and the source, provide a link to the Creative Commons licence, and indicate if changes were made. The images or other third party material in this article are included in the article's Creative Commons licence, unless indicated otherwise in a credit line to the material. If material is not included in the article's Creative Commons licence and your intended use is not permitted by statutory regulation or exceeds the permitted use, you will need to obtain permission directly from the copyright holder. To view a copy of this licence, visit http://creativecommons.org/licenses/by/4.0/.

\section{References}

1. World Health Organization. Injuries and violence: the facts. WHO. 2012. https://www.who.int/violence_injury_prevention/ publications/factsheets/all/en/. Accessed 31 Oct 2021.

2. Abu Dhabi Health Statistics 2017. https://www.doh.gov.ae/-/ media/Feature/Resources/AbuDhabiHealthStatistics.ashx. Accessed 31 Oct 2021.

3. DiFazio LT, Curran T, Bilaniuk JW, Adams JM, Durling-Grover R, Kong K, Nemeth ZH. The impact of the COVID-19 pandemic on hospital admissions for trauma and acute care surgery. Am Surg. 2020;86:901-3.

4. Nunez JH, Sallent A, Lakhani K, Guerra-Farfan E, Vidal N, Ekhtiari S, Minguell J. Impact of the COVID-19 pandemic on an emergency traumatology service: experience at a Tertiary Trauma Centre in Spain. Injury. 2020;51:1414-8.

5. Kamine TH, Rembisz A, Barron RJ, Baldwin C, Kromer M. Decrease in trauma admissions with COVID-19 pandemic. West J Emerg Med. 2020;21:819-22.

6. Wong JSH, Cheung KMC. Impact of COVID-19 on orthopaedic and trauma service: an epidemiological study. J Bone Jt Surg Am. 2020;102:e80.

7. Yasin YJ, Alao DO, Grivna M, Abu-Zidan FM. Impact of the COVID-19 pandemic on road traffic collision injury patterns and severity in Al-Ain City, United Arab Emirates. World J Emerg Surg. (In press).

8. Yasin YJ, Grivna M, Abu-Zidan FM. Global impact of COVID19 pandemic on road traffic collisions. World J Emerg Surg. $2021 ; 16: 51$

9. Statistics yearbook of Abu Dhabi. https://www.scad.gov. ae/Release\% 20Documents/Statistical\%20Yearbook\%20of\% 20Abu\%20Dhabi_2020_Annual_Yearly_en.pdf. Accessed 31 Oct 2021.

10. Grivna M, Aw TC, El-Sadig M, et al. The legal framework and initiatives for promoting safety in the United Arab Emirates. Int J Inj Control Saf Promot. 2012;19:278-89.

11. Alao DO, Cevik AA, Eid HO, Jummani Z, Abu-Zidan FM. Trauma system developments reduce mortality in hospitalized trauma patients in Al-Ain City, United Arab Emirates, despite increased severity of injury. World J Emerg Surg. 2020;15:49.

12. Cevik AA, Alao DO, Eid HO, Grivna M, Abu-Zidan FM. Current changes in the epidemiology of fall-related injuries in Al Ain City, United Arab Emirates. PLoS One. 2021;16:e0257398.

13. Zsilavecz A, Wain H, Bruce JL, et al. Trauma patterns during the COVID-19 lockdown in South Africa expose vulnerability of women. S Afr Med J. 2020;110:1110-2.

14. Abdallah $\mathrm{HO}$, Zhao $\mathrm{C}$, Kaufman E, et al. Increased firearm injury during the COVID-19 pandemic: a hidden urban burden. J Am Coll Surg. 2021;232(2):159-168.e3.

15. Adiamah A, Thompson A, Lewis-Lloyd C, et al. The ICON Trauma Study: the impact of the COVID-19 lockdown on major trauma workload in the UK. Eur J Trauma Emerg Surg. 2021;47:637-45.

16. Moonesar IA, Hussain M, Gaafar R, Suliman D. Rapid response: informing United Arab Emirates' response to the COVID-19 pandemic. 2020. https://www.researchgate.net/publication/34156 6734_Rapid_Response_Informing_United_Arab_Emirates' Response_to_the_COVID-19_Pandemic. Accessed 31 Oct 2021.

17. Salottolo K, Caiafa R, Mueller J, et al. Multicenter study of US trauma centres examining the effect of the COVID-19 pandemic 
on injury causes, diagnoses and procedures. Trauma Surg Acute Care Open. 2021;6:e00655.

18. Rajput K, Sud A, Rees M, Rutka O. Epidemiology of trauma presentations to a major trauma centre in the North West of England during the COVID-19 level 4 lockdown. Eur J Trauma Emerg Surg. 2021;47:631-6.

19. Jefferies O, Kealey D, Yoong S, Houston R, Tennyson C. The effect of the COVID-19 pandemic on the workload of an adult major trauma centre in Northern Ireland. Ulst Med J. 2021;90:13-5.

20. Waseem S, Nayar SK, Hull P, Carrothers A, Rawal J, Chou D, Khanduja V. The global burden of trauma during the COVID19 pandemic: a scoping review. J Clin Orthop Trauma. 2021;12:200-7.

21. Riuttanen A, Ponkilainen V, Kuitunen I, Reito A, Sirola J, Mattila VM. Severely injured patients do not disappear in a pandemic: incidence and characteristics of severe injuries during COVID-19 lockdown in Finland. Acta Orthop. 2021;92:249-53.

22. Henry N, Parthiban S, Farroha A. The effect of COVID-19 lockdown on the incidence of deliberate self-harm injuries presenting to the emergency room. Int J Psychiatry Med. 2021;56:266-77.

23. Ratnasekera AM, Seng SS, Jacovides CL, Kolb R, Hanlon A, Stawicki SP, Martin ND, Kaufman EJ. Rising incidence of interpersonal violence in Pennsylvania during COVID-19 stay-at home order. Surgery. 2022 Feb;171(2):533-540. https://doi.org/ 10.1016/j.surg.2021.06.024. Epub 2021 Jun 23. PMID: 34294449; PMCID: PMC8782280.

24. Sheek-Hussein M, Abu-Zidan FM, Stip E. Disaster management of the psychological impact of the COVID-19 pandemic. Int J Emerg Med. 2021;14:19. 